1. May, C., et al. 2000. Therapeutic haemoglobin synthesis in beta-thalassaemic mice expressing lentivirus-encoded human beta-globin. Nature. 406:82-86.

2. Pawliuk, R., et al. 2001. Correction of sickle cell disease in transgenic mouse models by hematopoietic gene therapy. Science. 294:2368-2371.

3. Moscow, J.A., et al. 1999. Engraftment of MDR1 and NeoR gene-transduced hematopoietic cells after breast cancer chemotherapy. Blood. 94:52-61.

4. Abonour, R., et al. 2000. Efficient retrovirusmediated transfer of the multidrug resistance 1 gene into autologous human long-term repopulating hematopoietic stem cells. Nat. Med. 6:652-658.

5. Allay, J.A., Dumenco, L.L., Koc, O.N., Liu, L., and
Gerson, S.L. 1995. Retroviral transduction and expression of the human alkyltransferase cDNA provides nitrosourea resistance to hematopoietic cells. Blood. 85:3342-3351.

6. Allay, J.A., Davis, B.M., and Gerson, S.L. 1997. Human alkyltransferase-transduced murine myeloid progenitors are enriched in vivo by BCNU treatment of transplanted mice. Exp. Hematology. 25:1069-1076.

7. Gerson, S.L. 2002. Clinical relevance of MGMT in the treatment of cancer. J. Clin. Oncol. 20:2388-2399.

8. Persons, D.A., et al. 2003. Successful treatment of murine beta-thalassemia using in vivo selection of genetically modified, drug-resistant hematopoietic stem cells. Blood. 102:506-513.

9. Neff, T., et al. 2003. Methylguanine methyltransferase-mediated in vivo selection and chemoprotection of allogeneic stem cells in a large-animal model. J. Clin. Invest. 112:1581-1588. doi:10.1172/JCI200318782.

10. Zielske, S.P., et al. 2003. In vivo selection of MGMT(P140K) lentivirus-transduced human NOD/SCID repopulating cells without pretransplant irradiation conditioning. J. Clin. Invest 112:1561-1570. doi:10.1172/JCI200317922.

11. Kaiser, J. 2003. Gene therapy: seeking the cause of induced leukemias in X-SCID trial. Science. 299:495.

12. Friedmann, T. 2003. Gene therapy's new era: a balance of unequivocal benefit and unequivocal harm. Mol. Ther. 8:5-7.

13. Kohn, D.B., et al. 2003. American Society of Gene Therapy (ASGT) ad hoc subcommittee on retroviral-mediated gene transfer to hematopoietic stem cells. Mol. Ther. 8:180-187.

\title{
Therapeutic CD154 antibody for lupus: promise for the future?
}

\section{Garnett Kelsoe}

Department of Immunology, Duke University, Durham, North Carolina, USA

\begin{abstract}
Systemic lupus erythematosus (SLE) is a prototypical systemic autoimmune disease characterized by the production of pathogenic autoantibodies. A new study (see the related article beginning on page 1506) demonstrates that passive antibody specific for the TNF family member, CD154, ameliorates disease by reducing levels of self-reactive antibody in the serum. This study demonstrates a substantial potential for antiCD154 antibody in the treatment of humoral autoimmunity.
\end{abstract}

J. Clin. Invest. 112:1480-1482 (2003). doi:10.1172/JCI200320371.

The autoimmune disease systemic lupus erythematosus (SLE) is characterized by the production of highaffinity IgG antibodies against double-stranded DNA (dsDNA). Typically, these pathogenic autoantibodies are encoded by mutated I $g$ genes, and the frequency and pattern of mutations strongly suggest that the source of autoreactive antibodies is B cells that have participated in the germinal

Address correspondence to: Garnett Kelsoe, Department of Immunology, Duke University Medical Center 3010, Duke University, Durham, North Carolina 27710, USA. Phone: (919) 613-7815; Fax: (919) 613-7878; E-mail: ghkelsoe@duke.edu.

Conflict of interest: The author has declared that no conflict of interest exists.

Nonstandard abbreviations used: systemic lupus erythematosus (SLE); double-stranded DNA (dsDNA); germinal center (GC); follicular dendritic cell (FDC); dark zone (DZ); light zone (LZ); activation-induced cytidine deaminase (AID); somatic hypermutation (SHM); class switch recombination (CSR); SLE disease activity index (SLEDAI). center (GC) reaction. Indeed, GCs appear spontaneously in many strains of lupus-prone mice (1), and extraordinary numbers of GC B cells and their progeny are present in the blood of SLE patients (2).

GCs are organized collections of antigen-activated $\mathrm{T}$ and $\mathrm{B}$ lymphocytes in secondary lymphoid tissues (3) or sites of chronic inflammation $(4,5)$. Although transient GC-like reactions can be elicited by thymusindependent pathways (6-8), most $\mathrm{GC}$ responses are thymus-dependent. GC B cells require ongoing survival and proliferation signals that depend on CD154-CD40 signaling (9). CD154, a member of the TNF family, is inducibly expressed on the surface of CD4 T lymphocytes, whereas its ligand, CD40, is constitutively present on B lymphocytes (reviewed in ref. 10).

$\mathrm{T}$ cell-dependent GCs begin with the separate activation of $\mathrm{T}$ and $\mathrm{B}$ cells by antigen (11). In secondary lym- phoid tissues, binding of antigen modifies B cell chemotaxis, resulting in migration toward the $\mathrm{T}$ cell zone (12), where cognate T cell-B cell interaction expands both lymphocyte populations. Soon afterward, activated T and $\mathrm{B}$ cells emigrate from $\mathrm{T}$ cell areas into the reticula of follicular dendritic cells (FDCs) that define the B cell follicle (Figure 1). The immigrant $\mathrm{B}$ lymphocytes proliferate in the FDC reticulum to generate nascent GCs and, in the process, acquire distinctive phenotypes, including expression of the CD69 activation antigen and several differentiation markers, including CD38 and CD27. Remarkably, human $\mathrm{CD}^{3} 8^{+} \mathrm{GC}$ B cells can also express variable levels of CD154, especially under conditions of chronic stimulation $(13,14)$, and appear to have the capacity for continued self-activation.

GCs become polarized into histologic dark and light zones (DZ, LZ). The $\mathrm{DZ}$ is proximal to the $\mathrm{T}$ cell area and contains rapidly dividing $B$ cells called centroblasts that express little or no surface immunoglobulin. The more distal LZ contains the bulk of the activated FDC network, antigen-specific CD4 T cells, and nondividing B cells known as centrocytes. Centrocytes express surface immunoglobulin and are thought to be the progeny of $\mathrm{DZ}$ centroblasts. In turn, selected centrocytes likely reenter the DZ and regain the centroblast form.

The enzyme AID (activation-induced cytidine deaminase) drives both somatic hypermutation (SHM) and immunoglobulin class switch recombination (CSR) in GC B cells (15). SHM introduces point mutations and occasional small deletions into the 


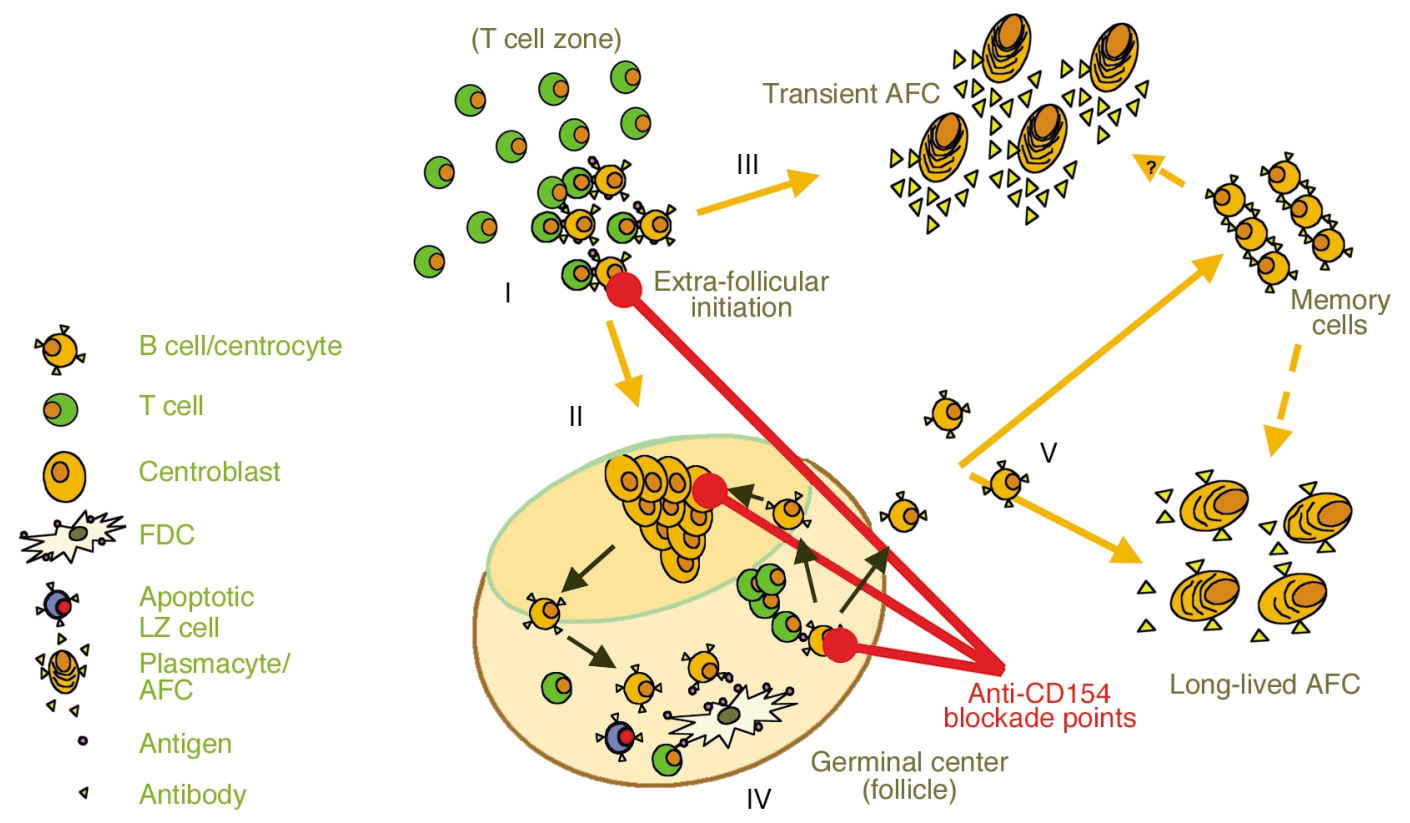

\section{Figure 1}

Potential cellular targets for passive CD154 antibody to interrupt thymus-dependent GC and antibody responses. Antigen-specific T and $B$ lymphocytes meet at the interface of $T$ and $B$ cell zones (follicles) in secondary lymphoid tissues (I). CD40-CD154 interaction results in the local proliferation of both lymphocyte types. Following clonal expansion, a fraction of activated T and B cells, prompted by CD40CD154 signaling, migrate into the follicle to initiate the GC reaction (II). In GCs, B cells proliferate and activate AID-dependent SHM and CSR. Other clonally related B lymphocytes are retained in extra-follicular sites and differentiate into antibody-forming cells (AFCs), or plasmacytes (III). These transient AFCs do not express AID and do not support CSR and SHM. In GCs, centroblasts proliferate in the $D Z$, migrate to the $L Z$ as centrocytes, present antigen to $L Z T$ cells, and receive signals that direct either their exit from the GC or return to the DZ (IV). Centrocytes that do not receive survival signals die by apoptosis. Active GCs require continuous CD40-CD154 signaling; these signals may represent homotypic interaction between $C D 40^{+} \mathrm{CD} 154^{+}$centroblasts or heterotypic signals between $\mathrm{CD} 40^{+}$centrocytes and CD154 ${ }^{+} \mathrm{CD} 4 \mathrm{~T}$ cells. Selected B cells exit the GC (V) to enter the memory or long-lived plasmacyte compartments. Grammer et al. (17) observe that passive anti-CD154 reduces the numbers of IgD $\mathrm{C}^{+} \mathrm{CD} 38^{+} \mathrm{GC}$ precursors (I and II) and CD38 bright plasmacytes (III and $\mathrm{V})$ but has little effect on circulating GC $\left(\operatorname{IgD}-\mathrm{CD} 38^{+}\right)$cells (IV). The primary effect(s) of CD154 antibody in SLE patients may be to interrupt the early initiation (I) and/or migrations steps (II) of the GC reaction.

$\mathrm{V}(\mathrm{D}) \mathrm{J}$ regions of transcriptionally active immunoglobulin genes. Mutations are probably introduced in centroblasts and accumulate during repeated rounds of centroblast/centrocyte migration between the DZs and LZs (Figure 1). Intense selection for higher-affinity mutants establishes GCs as foci of rapid somatic evolution and antibody affinity maturation. CSR ensures that mutated, high-affinity antibodies acquire various effector activities and can be delivered throughout the body.

With time, centrocytes leave GCs and enter into at least two phenotypically distinct compartments of memory cells (Figure 1). One compartment, comprising antigen-specific, small, resting B lymphocytes, defines the classical memory B lymphocyte. The other contains long-lived plasmacytes that secrete high-affinity antibody and are capable of maintaining significant levels of serum antibody for years, if not decades. Characteristically, fully differentiated human plasmacytes express high levels of CD38.

\section{Passive CD154 antibody therapy for lupus}

In this issue of the JCI, Amrie Grammer and colleagues demonstrate that administration of a humanized monoclonal antibody specific for CD154 (16) produced significant clinical benefit in a small group of active SLE patients (17). Anti-CD154 promptly decreased levels of dsDNA serum autoantibody and also diminished the numbers of GC B cells and plasmacytes circulating in the blood. While dsDNA antibody levels rebounded after treatment, other objective measures of disease including proteinuria and scores on a standardized index of SLE activity (SLEDAI) remained significantly suppressed over a 20 -month post-treatment period. These promising results are tempered by the fact that this clinical study was prematurely ended due to adverse side effects (16). Nonetheless, the substantial clinical improvements observed by Grammer et al. suggest a therapeutic strategy of considerable potential. But how, and where, does anti-CD154 antibody ameliorate SLE?

\section{Potential cell targets for anti-CD154}

There are at least three stages of humoral immune responses in which interruption of CD154-CD40 signals can abrogate the GC reaction and antibody responses (Figure 1). AntiCD154 can (i) interrupt the initial, cognate interaction between antigen- 
specific $\mathrm{T}$ and $\mathrm{B}$ lymphocytes within and/or adjacent to the $T$ cell areas of lymph nodes or spleen. Blockade of this interaction prevents both the formation of GCs and the initial production of plasmacytes. Later in the response, anti-CD154 disrupts GCs either by (ii) preventing homotypic CD154-CD40 interaction among proliferating DZ centroblasts; or (iii) interfering with collaboration between LZ T cells and centrocytes. Of note, Grammer et al. (17) find that anti-CD154 elicits relatively little change in the numbers of $\mathrm{IgD}^{-} \mathrm{CD} 38^{+}$ blood cells in SLE patients but rather causes substantial reductions in the numbers of $\mathrm{IgD}^{+} \mathrm{CD} 38^{+}$cells and CD38 ${ }^{\text {high }}$ plasmacytes. Reductions in these pre-switch GC cells and plasmacytes correlate with reductions in autoantibody titers and DNA-specific blood plasmacytes (16). The biased effects on pre-switch cells and plasmacytes suggests that the primary benefit of anti-CD154 treatment in SLE may be the disruption of plasmacyte generation/differentiation, perhaps at a point before B cells become fully differentiated into centroblasts or centrocytes.

Even though passive CD154 antibody did not reduce the number of $\mathrm{IgD}^{-} \mathrm{CD} 38^{+} \mathrm{GC}$ cells in the blood of SLE patients, the ability to abrogate active GC responses remains an attractive feature of this therapy (17). In SLE, IgG autoantibodies against dsDNA appear to descend from B cell clones that do not react to DNA. These pathogenic autoantibodies arise by SHM, not in response to DNA but to some other antigen or by some nonantigenic stimulus that supports SHM. In bone marrow, autoreactive cells generated during B lymphocyte development are deleted by apoptosis (18), enfeebled (19), or given a new reactivity by receptor editing (20). SHM in GC B cells must occasionally produce autoreactive antigen-receptors absent from the tolerizing environment of the bone marrow. Although GCs may possess the capacity to remove self-reactive lymphocytes by apoptosis (21), this process does not appear particularly efficient (22). Thus, anti-CD154 therapy may carry the additional benefit of halting the generation of autoreactive B cells or their affinity maturation into pathogenic forms.

Regardless of where, or even how, passive antibody to CD154 acts to reduce SLE disease, Grammer and colleagues have taken an important step toward defining the promise of CD154-CD40 interaction as a target of therapy (17). The future is not clear - the antibody used in these studies elicited unacceptable side effects but the direction of study has been clearly defined. If different autoimmune diseases represent distinct errors of immune regulation, analogous approaches to other therapeutic targets are surely in store.

1. Luzina, I.G., et al. 2001. Spontaneous formation of germinal centers in autoimmune mice. J. Leukoc. Biol. 70:578-584.

2. Arce, E., et al. 2001. Increased frequency of pregerminal center B cells and plasma cell precursors in the blood of children with systemic lupus erythematosus. J. Immunol. 167:2361-2369.

3. Kelsoe, G. 1996. Life and death in germinal centers (redux). Immunity. 4:107-111.

4. Stott, D.I., Hiepe, F., Hummel, M., Steinhauser, G., and Berek, C. 1998. Antigen-driven clonal proliferation of $\mathrm{B}$ cells within the target tissue of an autoimmune disease. The salivary glands of patients with Sjogren's syndrome. J. Clin. Invest. 102:938-946.

5. Kim, H.J., Krenn, V., Steinhauser, G., and Berek, C. 1999. Plasma cell development in synovial germinal centers in patients with rheumatoid and reactive arthritis. J. Immunol. 162:3053-3062.
6. de Vinuesa, C.G., et al. 2000. Germinal centers without T cells. J. Exp. Med. 191:485-494.

7. Toellner, K.M., et al. 2002. Low-level hypermutation in T cell-independent germinal centers compared with high mutation rates associated with $\mathrm{T}$ cell-dependent germinal centers. J. Exp. Med. 195:383-389.

8. Lentz, V.M., and Manser, T. 2001. Cutting edge: germinal centers can be induced in the absence of T cells. J. Immunol. 167:15-20.

9. Han, S.K., et al. 1995. Cellular interaction in germinal centers. Roles of CD40 ligand and B7-2 in established germinal centers. J. Immunol. 155:556-567.

10. Foy, T.M., Aruffo, A., Bajorath, J., Buhlmann, J.E. and Noelle, R.J. 1996. Immune regulation by CD40 and its ligand GP39. Annu. Rev. Immunol. 14:591-617.

11. Garside, P., et al. 1998. Visualization of specific B and $\mathrm{T}$ lymphocyte interactions in the lymph node. Science. 281:96-99.

12. Reif, K., et al. 2002. Balanced responsiveness to chemoattractants from adjacent zones determines B-cell position. Nature. 416:94-99.

13. Grammer, A.C., McFarland, R.D., Heaney, J., Darnell, B.F., and Lipsky, P.E. 1999. Expression, reg ulation, and function of B cell-expressed CD154 in germinal centers. J. Immunol. 163:4150-4159.

14. Desai-Mehta, A., Lu, L., Ramsey-Goldman, R. and Datta, S.K. 1996. Hyperexpression of CD40 ligand by $\mathrm{B}$ and $\mathrm{T}$ cells in human lupus and its role in pathogenic autoantibody production. J. Clin. Invest. 97:2063-2073.

15. Muramatsu, M., et al. 2000. Class switch recom bination and hypermutation require activationinduced cytidine deaminase (AID), a potential RNA editing enzyme. Cell. 102:553-563.

16. Boumpas, D.T., et al. 2003. A short course of BG9588 (anti-CD40 ligand antibody) improves serologic activity and decreases hematuria in patients with proliferative lupus glomerulonephritis. Arthritis Rheum. 48:719-727.

17. Grammer, A.C., et al. 2003. Abnormal germinal center reactions in systemic lupus erythematosus demonstrated by blockade of CD154-CD40 interactions. J. Clin. Invest. 112:1506-1520. doi:10.1172/JCI200319301.

18. Hartley, S.B., et al. 1993. Elimination of self-reactive B lymphocytes proceeds in two stages: arrested development and cell death. Cell. 72:325-335.

19. Goodnow, C.C., et al. 1988. Altered immunoglobulin expression and functional silencing of selfreactive $B$ lymphocytes in transgenic mice. Nature. 334:676-682.

20. Nemazee, D., and Weigert, M. 2000. Revising B cell receptors. J. Exp. Med. 191:1813-1817.

21. Han, S., Zheng, B., Dal Porto, J., and Kelsoe, G. 1995. In situ studies of the primary immune response to (4-hydroxy-3- nitrophenyl)acetyl. IV. Affinity-dependent, antigen-driven B cell apoptosis in germinal centers as a mechanism for maintaining self- tolerance. J. Exp. Med. 182:1635-1644.

22. Reed, A.J., Riley, M.P., and Caton, A.J. 2000. Virusinduced maturation and activation of autoreactive memory B cells. J. Exp. Med. 192:1763-1774. 